\title{
Desenvolvimento ponderal na estimativa de peso vivo em ovinos da raça Poll Dorset*
}

\section{Growth rate in live weight estimation in Poll Dorset sheep breed}

\author{
Diego de Azevedo Mota. ${ }^{* *}$ Bruna Laurindo Rosa, ${ }^{* *}$ Márcia Maria Oziemblowski, ${ }^{* * *}$ Thiago Vasconcelos Melo, ${ }^{* *}$ \\ Daniel Marino Guedes de Carvalho*****
}

\begin{abstract}
Resumo
Objetivou-se avaliar nesse estudo o uso da biometria durante o crescimento de cordeiros da raça Poll Dorset, com o intuito de estimar o peso vivo. Foram utilizados 32 cordeiros, sendo 20 machos e 12 fêmeas, nascidos entre julho e setembro de 2014 e 2015 , sendo as coletas realizadas do nascimento aos 165 dias com intervalo de 15 dias entre as avaliações. O estudo gerou 1920 observações de pesos e medidas biométricas. A cada tomada de peso também foram realizadas medidas de perímetro torácico, com a utilização de fita métrica graduada em centímetros, comprimento de corpo, altura de cernelha e altura de garupa utilizando um hipômetro. Os dados foram submetidos à análise de correlação e a partir destes gerou-se gráficos de dispersão e estimaramse equações de regressão, além de estimar o desenvolvimento ponderal. O perímetro torácico $(0,88)$ foi a característica mais associada ao peso corporal in vivo. As equações de regressão reafirmaram a alta relação entre perímetro torácico e peso vivo, que foi de $94 \%$. O desenvolvimento ponderal caracterizou animais com padrão racial homogêneo. Conclui-se que o perímetro torácico está altamente associado ao peso corporal e que equações de regressão geradas a partir de medidas biométricas servem para estimar o peso corporal de ovinos da raça Poll Dorset.
\end{abstract}

Palavras-chave: biometria, perímetro torácico, altura de cernelha, altura de garupa.

\begin{abstract}
The objective of this work was evaluated the growth lambs Poll Dorset to estimate the live weight. Were used 32 lambs, 20 males and 12 females, born between July and September 2014 and 2015, and the assessment carried out from birth to 165 days with an interval of 15 days between assessments. The study generated 1920 observations of weights and biometric measurements. Each weight measurement were also performed thoracic measures with tape graduated in centimeters, body length and hip height using a hipometer. The data was submitted to correlation analysis and generated scatter plots and regression equations estimating the weight development. The thoracic measures $(0.88)$ was the characteristic most associated with body weight in vivo. The regression equations reaffirmed the high relationship between thoracic measures and body weight, which was $94 \%$. The weight development featured animals with homogeneous racial pattern. We conclude that the knowledge of the weight development reflects a racial standard and the thoracic measures is highly associated with body weight and regression equations generated from this measurements are used to estimate the body weight of the breed sheep Poll Dorset.
\end{abstract}

Keywords: biometric, heart girth, withers height, hip height.

\section{Introdução}

A realização do controle zootécnico é indispensável e deve ser implantado de imediato no rebanho a fim de que a atividade pecuária se torne rentável. O desenvolvimento ponderal, ou seja, as variações de peso durante a vida do animal é uma medida muito importante. Porém nem sempre o ovinocultor dispõe de balança na propriedade e, para contornar o problema, podese fazer o uso da biometria ou barimetria, que é uma técnica simples, funcional, acessível, pela qual se consegue estimar o peso vivo através de medidas corporais, como perímetro torácico, comprimento, entre outras.

De acordo com Pinheiro et al. (2007), estudos comparativos da biometria em ovinos permitem análises entre tipos raciais, pesos e manejo nutricional, sendo um método prático e de baixo custo. Mesmo que medidas morfológicas isoladas não sejam suficientes para caracterizar animais in vivo e carcaças, o uso combinado entre elas permitem melhores ajustes e comparações entre outras características dos animais. Como exemplo, o comprimento do corpo, que reflete maior área de

\footnotetext{
*Recebido em 8 de março de 2016 e aceito em 4 de março de 2018.

**Universidade Federal dos Vales do Jequitinhonha e Mucuri, Instituto de Ciências Agrárias - ICA, Unaí, Minas Gerais, Brasil.

*** Universidade Federal do Acre (UFAC), Centro de Ciências Biológicas e da Natureza (CCBN), Rio Branco, Acre, Brasil.

****Universidade Federal da Fronteira Sul (UFFS), Campus de Erechim, Rodovia ERS 135, km 72, Interior de Erechim -RS.

*****Universidade Federal do Mato Grosso, Instituto de Ciências Exatas e da Terra - ICET, Barra do Garças - Mato Grosso, Brasil. Autor para correspondência: E-mail: diegomota@zootecnista.com.br
} 
olho de lombo, ou então o perímetro torácico que tem relação direta com a capacidade cardiorrespiratória. O acompanhamento do desenvolvimento ponderal de um rebanho é importante para os manejos nutricional, reprodutivo e sanitário e a forma mais comum de se conhecer o peso vivo é através da pesagem.

Existem poucos estudos em relação a estimativa de peso em ovinos, principalmente para o grupo genético ou raça em questão (Poll Dorset), a qual se apresenta como uma raça prolífica, dócil, que cicla o ano inteiro, com boa qualidade de carcaça (comprida, light e precoce) e acentuada habilidade materna e fertilidade, partos múltiplos, rusticidade e alta conversão alimentar. Neste sentido o presente trabalho tem por objetivo avaliar o desenvolvimento ponderal e verificar o quanto significantes são as estimativas de peso vivo obtidas através de medidas biométricas de ovinos Poll Dorset, quando comparadas ao peso mensurado com balança.

\section{Material e métodos}

O experimento foi conduzido na Cabanha Don Cortes, no município de Erechim, norte do estado do Rio Grande do Sul, a uma altitude de $783 \mathrm{~m}$, latitude $27^{\circ} 62^{\prime} 65^{\prime \prime}$ Sul e longitude $52^{\circ}$ 24' 04" Oeste. De acordo com a escala de Koopen o município está localizado na Zona Climática fundamental temperada $(C)$, apresentando clima do tipo fundamental úmido (f) e variedade específica subtropical (Cfa). O clima local é descrito como subtropical úmido ( $\mathrm{Cfa}$ ), com chuva bem distribuída durante o ano.

Foram utilizados 32 cordeiros puros de origem, da raça Poll Dorset, sendo 20 machos e 12 fêmeas, divididos nas duas estações de nascimento, que foram de julho a setembro, dos anos de 2014 e 2015. Em ambos os anos, as ovelhas e seus respectivos cordeiros foram submetidos a um sistema semiextensivo de manejo. A alimentação das mães era constituída de pastagem perene de capim-Vaquero (Cynodon dacylon), feno de azevém (Lolium multiflorum) ou feno de Tifton (Cynodon spp) no período da tarde e uma suplementação de concentrado (18\% PB) 600g/cabeça/dia, divididos no período da manhã e tarde. Para os cordeiros o período de acesso ao creep feeding se deu em torno dos 20 dias de vida, onde tiveram acesso a um concentrado (22\% $\mathrm{PB}$ ) a uma quantidade equivalente a $1,0 \%$ do peso vivo, nos períodos da manhã e tarde, além de feno de azevém (Lolium multiflorum) ou feno de Tifton (Cynodon spp) no período da tarde. O desmame ocorreu entre 60 e 90 dias, de acordo com a condição corporal individual.

Após o desmame os cordeiros passaram a um sistema de confinamento em baias separadas para machos e fêmeas. Os animais foram monitorados quanto à infecção helmíntica através da técnica de Famacha, conforme Molento et al. (2004).

A coleta de dados se deu sistematicamente do nascimento aos 165 dias, a um intervalo de 15 dias. No período de aproximadamente 5 meses e meio foram feitas 12 coletas de dados por animal, resultando em 1920 observações de pesos e medidas biométricas dos cordeiros.

Segundo a metodologia adaptada de Souza et al. (2014), as características analisadas foram o perímetro torácico (PT) que é o perímetro imediatamente caudal á escápula passando pelo esterno e pelos processos espinhais das vértebras torácicas; comprimento do corpo (CC), linha reta entre a articulação escápulo-umeral á tuberosidade coxal do ísquio; altura de cernelha (AC), que é a distância medida a partir da região caudal da cernelha, traçando-se uma perpendicular até ao solo), altura da garupa $(A G)$ que é a distância medida do solo à tuberosidade sacral do ílio estando os animais em superfície plana e bem aprumados e por fim o peso corporal dos animais.

Para a pesagem dos cordeiros até os $25 \mathrm{~kg}$ foi usado balança tipo relógio com capacidade de até 25 kg e graduação mínima de $100 \mathrm{~g}$. Para pesagem dos cordeiros com mais de $25 \mathrm{~kg}$ foi utilizado balança analógica com precisão de $100 \mathrm{~g}$ e com capacidade para $300 \mathrm{~kg}$. A aferição das medidas biométricas de natureza circular, como o perímetro torácico foram mensuradas com o auxílio de fita métrica flexível graduada em centímetros. Já as mensurações de natureza linear, como o comprimento e alturas de cernelha e garupa foram realizadas com o auxílio de um hipômetro, sendo que este instrumento consiste em uma régua graduada, associada a dois apoios de medição, um fixo à régua e o outro ao cursor que, com um mínimo de folga, desliza sobre a régua graduada permitindo realizar a medida da distância entre dois pontos definidos.

Os resultados foram submetidos à análise de correlações de Pearson, com $5 \%$ de probabilidade, pelo procedimento CORR (SAS, 2001). Os resultados obtidos na correlação de cada medida biométrica com o PV foram comparados entre si e interpretados em forma de gráficos de dispersão. A partir das correlações observadas foram estimadas equações de regressão tendo como variável dependente o peso corporal.

\section{Resultados e discussão}

As medidas zoométricas dos cordeiros Poll Dorset ficam evidenciadas na (Tabela 1) onde é possível visualizar a média inicial e final do peso e das medidas biométricas, além dos valores mínimos e máximos dos mesmos.

Tabela 1: Valores médios, mínimos e máximos iniciais e finais de peso e medidas biométricas de ovinos Poll Dorset

\begin{tabular}{clccc}
\hline Variáveis & & Média & Mínimo & Máximo \\
\hline & Inicial & 4,60 & 2,70 & 5,50 \\
Peso Corporal $(\mathrm{kg})$ & Final & 50,80 & 40,50 & 61,50 \\
& Inicial & 33,40 & 29,00 & 37,00 \\
Comprimento Corporal (cm) & Final & 74,00 & 68,00 & 81,00 \\
& Inicial & 39,10 & 33,00 & 43,00 \\
Perímetro Torácico $(\mathrm{cm})$ & Final & 82,30 & 76,00 & 87,00 \\
& Inicial & 38,90 & 34,00 & 43,00 \\
Altura Cernelha $(\mathrm{cm})$ & Final & 65,30 & 61,00 & 71,00 \\
& Inicial & 40,10 & 35,00 & 44,00 \\
Altura Garupa $(\mathrm{cm})$ & Final & 66,50 & 62,00 & 74,00 \\
\hline
\end{tabular}

$\mathrm{Na}$ (Tabela 2) verifica-se que o comprimento corporal (CC) apresentou média de 61,85, o que demonstra um tipo corporal longilíneo. Vargas Junior et al (2011) e Yamamoto et al.(2007) encontraram médias 49,8 e 58,03 cm para cordeiros Pantaneiros e ovinos cruzados Ile de France $X$ Ideal, respectivamente. Foi 
observado valor médio de perímetro torácico (PT) de $67,40 \mathrm{~cm}$, o que demonstra animais com boa capacidade cardiorrespiratória, implicando positivamente no desenvolvimento ponderal. Valores semelhantes foram encontrados por Silva et al. (2008) e que observaram valores médios próximos a $70,0 \mathrm{~cm}$ com cordeiros da raça Morada Nova. Lima et al. (2010) encontraram valores inferiores de $38,59 \mathrm{~cm}$ ao nascer e $67,8 \mathrm{~cm}$ aos 3 meses em ovinos Santa Inês.

Tabela 2: Valores médios, ganho médio diário (GMD), e desvios padrão, de comprimento corporal (CC), perímetro torácico $(\mathrm{PT})$, altura de cernelha $(\mathrm{AC})$, altura de garupa $(A G)$ e peso corporal $(P C)$ de cordeiros Poll Dorset

\begin{tabular}{cr}
\hline Variáveis & $\begin{array}{r}\text { Médias e Desenvolvimento } \\
\text { Ponderal }\end{array}$ \\
\hline Comprimento Corporal $(\mathrm{cm})$ & $61,85( \pm 4,20)$ \\
Aumento cm/dia & $0,25( \pm 0,03)$ \\
Perímetro Torácico $(\mathrm{cm})$ & $67,40( \pm 5,10)$ \\
Aumento cm/dia & $0,26( \pm 0,05)$ \\
Altura da Cernelha $(\mathrm{cm})$ & $56,80( \pm 1,40)$ \\
Aumento cm/dia & $0,16( \pm 0,04)$ \\
Altura da Garupa $(\mathrm{cm})$ & $57,61( \pm 1,60)$ \\
Aumento cm/dia & $0,16( \pm 0,04)$ \\
Peso Corporal $(\mathrm{kg})$ & $31,12( \pm 3,80)$ \\
GMD kg/dia & $0,28( \pm 0,09)$
\end{tabular}

A média da altura da cernelha (AC) e altura de garupa (AG) foram de 56,8 e $57,61 \mathrm{~cm}$, respectivamente. Costa Junior et al., (2006) ressaltaram que a obtenção das medidas de AC e AG são considerados indicativos de diferença na velocidade de crescimento ósseo em relação aos aumentos em peso na carcaça. Cunha Filho et al. (2010) em estudos com 25 carneiros Texel com 14 meses de idade encontrou uma média de 71,15 $\mathrm{cm}$ na AC.

O Ganho Médio Diário (GMD) foi de $280 \mathrm{~g} / \mathrm{dia}$, entre o nascimento até aos 165 dias de vida. Villarroel, et al. (2006), avaliando o GMD do nascimento até o desmame, foram de $85 \mathrm{e}$ 89 gramas e, do desmame ao peso final, foi de 63 e 80 gramas, respectivamente para os mestiços Santa Inês e Texel, sendo os animais mantidos em sistema semi intensivo de produção. Brochier e Carvalho, (2008) avaliando o GMD de cordeiros em confinamento sendo alimentados com diferentes teores de grão úmido de cervejaria, encontraram valores médios de $141 \mathrm{~g} / \mathrm{dia}$ no intervalo entre a desmama até o abate.

Os valores de peso e medidas biométricas evidenciadas nesse estudo demonstram diferenças com outros trabalhos. Essa discordância ocorre em função principalmente da variedade de tipos ou grupos genéticos e diferentes sistemas de produção. Rocha et al. (2003) coloca que em se tratando de animais cruzados e/ou mestiços não existe uma padronização do tamanho corporal. Desta forma, a comparação entre grupos genéticos que tenham um padrão racial semelhante, gerariam dados mais específicos e serviriam como ferramenta para a tomada de decisão em rebanhos homogêneos.

Correlações significativas foram observadas para todas as variáveis estudadas, sendo que todas foram de média a alta magnitude (Tabela 3). A característica altamente associada com o peso corporal (PC) foi o perímetro torácico $(r=0,88)$, seguido por associações médias para o comprimento de corpo $(r=$ $0,68)$, altura de garupa $(r=0,58)$ e altura de cernelha $(r=0,56)$. Diversos trabalhos (Santana et al. 2001; Ribeiro et al., 2004; Silva et al., 2006; Vargas Junior et al., 2011) têm demonstrado que existe alta correlação do peso vivo entre várias medidas corporais tais como: perímetro abdominal, perímetro de flanco, altura de cernelha, altura de garupa e comprimento corporal. Contudo, a medida corporal que apresenta maior correlação com o PC é o perímetro torácico (Silva et al., 2006; Vargas Junior et al., 2011). Os resultados demonstram que o perímetro torácico pode ser um bom indicador do peso corporal de ovinos.

Outra associação que merece destaque é entre a altura de garupa e altura de cernelha que apresentou o valor de 0,96. Esse valor demonstra que no momento de obtenção das medidas biométricas os animais estavam bem posicionados, com as quatro patas posicionadas em nível, visto que os animais da raça Poll Dorset apresentam a linha dorso-lombar retilínea, com o dorso, lombo e anca formando um plano largo e comprido, desde a cruz até a inserção da cola (Selaive e Osório, 2014).

Tabela 3: Correlações de Pearson entre peso corporal (PC) e medidas biométricas: comprimento de corpo (CC), perímetro torácico $(P T)$, altura da garupa $(A G)$, altura da cernelha $(\mathrm{AC})$ de ovinos da raça Poll Dorset

\begin{tabular}{lccccc}
\hline \multirow{2}{*}{ Variáveis } & \multicolumn{5}{c}{ Correlações } \\
\cline { 2 - 6 } & $\mathrm{PC}(\mathrm{kg})$ & $\mathrm{CC}(\mathrm{cm})$ & $\mathrm{PT}(\mathrm{cm})$ & $\mathrm{AC}(\mathrm{cm})$ & $\mathrm{AG}(\mathrm{cm})$ \\
\hline $\mathrm{PC}(\mathrm{kg})$ & 1,00 & $0,68^{*}$ & $0,88^{*}$ & $0,56^{*}$ & $0,58^{*}$ \\
$\mathrm{CC}(\mathrm{cm})$ & & 1,00 & $0,62^{*}$ & $0,71^{*}$ & $0,70^{*}$ \\
$\mathrm{PT}(\mathrm{cm})$ & & 1,00 & $0,67^{*}$ & $0,66^{*}$ \\
$\mathrm{AC}(\mathrm{cm})$ & & & 1,00 & $0,96^{*}$ \\
$\mathrm{AG}(\mathrm{cm})$ & & & & 1,00 \\
\hline
\end{tabular}

*Significativo $(P<0,05)$.

Os resultados mostraram que o acompanhamento de medidas biométricas pode ser ferramenta importante para o produtor na formação de lotes mais homogêneos, na tomada de decisões de manejo e na seleção de animais superiores, visto que estas informações podem ser um indicador do futuro potencial produtivo dos animais.

As equações de regressão e os coeficientes de determinação (R2) geradas para predizer o PC através das medidas biométricas PT, CC, AC e AG foram: $y=1,123 x-44,56,(0,944)$; $y=1,174 x-41,44,(0,919) ; y=1,689 x-64,82,(0,924)$ e $y=$ $1,676 x-65,43,(0,927)$, respectivamente (Figura 1). Sendo todas as equações fortemente confiáveis nas condições experimentais adotadas.

A importância de mais estudos relacionados a investigação científica, tanto na obtenção de dados de desenvolvimento ponderal, quanto na aquisição de correlações entre diferentes medidas biométricas com o peso corporal nas diversas raças de ovinos, sejam as mesmas lanadas ou deslanadas, podem num futuro próximo gerar a confecção de uma fita zootécnica específica para ovinos, proporcionando aos ovinocultores uma ferramenta de manejo que trará facilidades na tomada de decisões de rotina. 
Outro ponto que merece destaque sobre a importância das medidas corporais é que essas são de fundamental importância para a caracterização de um grupo genético e conhecimento do seu potencial para exploração comercial. As informações obtidas permitem a comparação entre rebanhos e contribui para a definição de um padrão racial para rebanhos de raças puras, servindo como referencial para programas de melhoramento genético (Cruz e Carneiro, 2003).

\section{Conclusões}

O perímetro torácico está altamente associado ao peso corporal. As demais medidas biométricas apresentam associações médias com o peso corporal.

Com base nos resultados obtidos, pode-se concluir que as equações de regressão geradas a partir das medidas biométricas servem para estimar o peso corporal de ovinos da raça Poll Dorset.

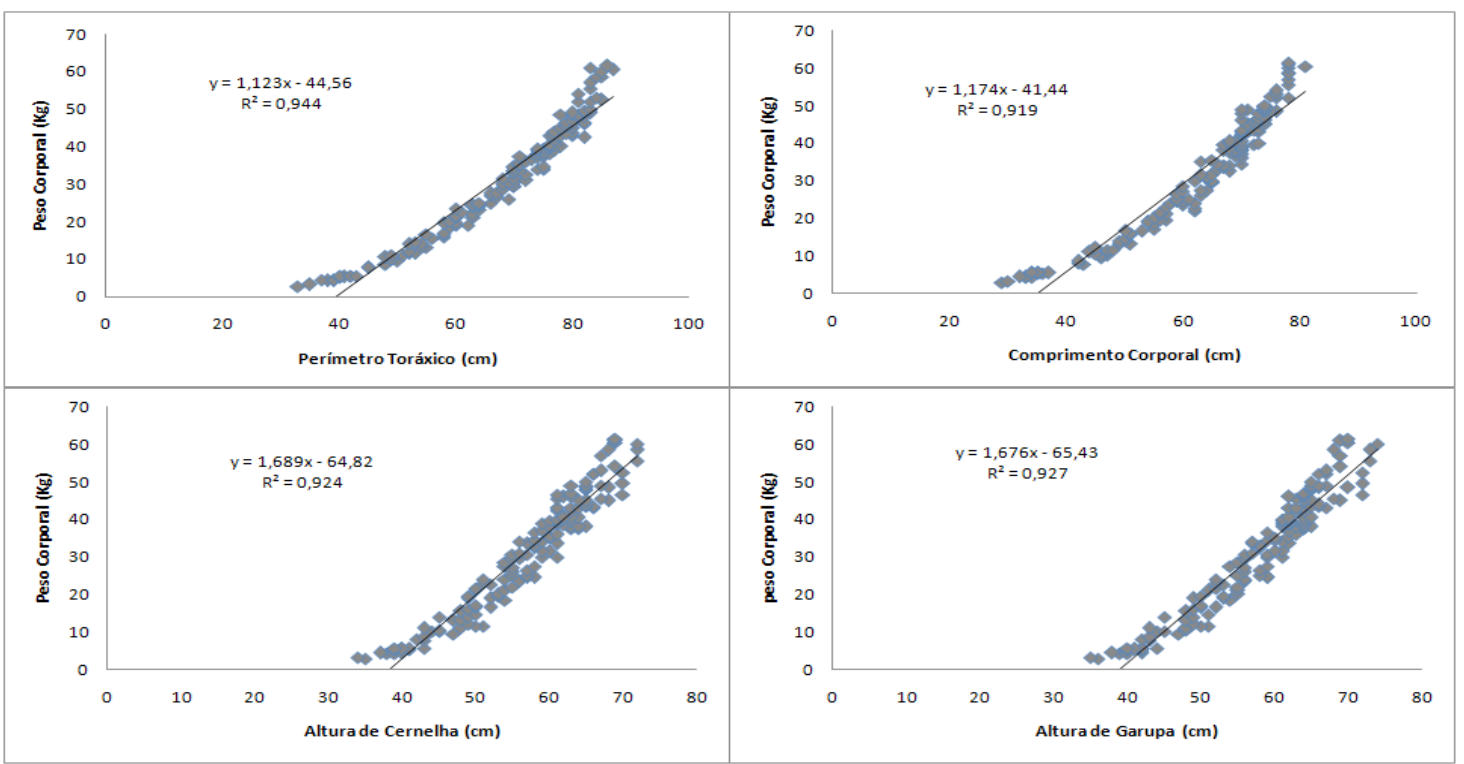

Figura 1: Gráficos de dispersão das equações obtidas a partir da correlação entre o peso vivo e medidas biométricas mensuradas de ovinos da raça Poll Dorset.

\section{Referências}

BROCHIER, M.A.; CARVALHO,S. Consumo, ganho de peso e análise econômica da terminação de cordeiros em confinamento com dietas contendo diferentes proporções de resíduo úmido de cervejaria. Arquivo Brasileiro de Medicina Veterinária e Zootecnia, v.60, n.5, p.1205-1212, 2008.

COSTA JÚNIOR, G.S.; CAMPELO, J.E.G.; AZEVÊDO, D.M.M.R.; MARTINS FILHO, R.; CAVALCANTE, R.R.; LOPES, J.B.;

OLIVEIRA, M.E. Caracterização morfométrica de ovinos da raça Santa Inês criados nas microrregiões de Teresina e Campo Maior, Piauí. Revista Brasileira de Zootecnia., v.35, n.6, p.2260-2267, 2006.

CRUZ, C.D; CARNEIRO, P.C.S. Modelos biométricos aplicados ao melhoramento genético. Viçosa: UFV, 2003. v.2. 585p.

CUNHA FILHO, L. F. C.; REGO, F. C. A; JUNIOR, F. A. B; STERZA, F. A. M; OKANO, W; TRAPP, S. M. Predição do peso corporal a partir de mensurações corporais em ovinos texel. Arquivos de Ciências Veterinárias e Zoologia, v.13, n.1, p. 5-7, 2010.

LIMA C.J.A., SANTOS A.D.F., OLIVEIRA V.S., MOURA C.N., CARVALHO C.T.G., JUNIOR A.M.F. Perímetro escrotal, peso e medidas corporais em ovinos da raça Santa Inês e mestiços Santa Inês x Dorper criados no sertão sergipano. In: $47^{\text {a Reunião }}$ Anual da Sociedade Brasileira de Zootecnia Salvador, BA, Anais... 2010. p.1 - 3.
MOLENTO, M.B.; TASCA, C.; GALLO, A.; FERREIRA, M.; BONONI, R.; STECCA, E. Método famacha como parâmetro clínico individual de infecção por "Haemonchus contortus" em pequenos ruminantes. Ciência Rural, v.34, n.4, p.1139-1145, 2004.

PINHEIRO, R.S.B.; SILVA SOBRINHO, A.G.; MARQUES, C.A.T.; YAMAMOTO, S.M. Biometria in vivo e da carcaça de cordeiros confinados. Archivos de Zootecnia, v.56, n.216, p.955-958. 2007.

RIBEIRO, N.L.1, A.N. MEDEIROS, RIBEIRO, M.N., PIMENTA FILHO E.C. Estimación del peso vivo de caprinos autóctonos brasileños mediante medidas morfométricas. Archives Zootecnia, v.53, n.203, p.341-344, 2004.

ROCHA, E.D.; ANDRADE, V.J; EUCLIDES FILHO, K.; NOGUEIRA, E.; FIGUEIREDO, G.R. Tamanho de vacas Nelore adultas e seus efeitos no sistema de produção de gado de corte. Arquivo Brasileiro de Medicina Veterinária e Zootecnia, v.55, n.4, p.474-9, 2003.

SANTANA, A. F. de; COSTA, G. B.; FONSECA, L. S. Correlações entre peso e medidas corporais em ovinos jovens da raça Santa Inês. Revista Brasileira de Saúde e Produção Animal, v.1, n.3, p.74-77, 2001.

SELAIVE, A.B.; OSÓRIO J. C. S. Produção de Ovinos no Brasil. Grupo Gem, 2014, p.656.

SILVA, D.C.; AZEVÊDO, D.M.M.R.; ALVES, A.A.; CAMPELO, J.E.G.; OLIVEIRA, M.E.; MALHADO, C.H.M. Estimativa do Peso Vivo Através do Perímetro Torácico de Ovinos Santa Inês. Revista Científica de Produção Animal, v.8, n.2, p.41-46. 2006 
SILVA, N.V.; COSTA, R.G.; MEDEIROS, A.N. Biometria e correlações com características de carcaça de cordeiros morada nova alimentados com dietas contendo feno de flor de seda. In: V CONGRESSO NORDESTINO DE PRODUÇÃO ANIMAL. Aracaju, SE. Anais... 2008. p.1-3.

SOUZA, D.S.; SILVA, H.P.; CARVALHO, J.M.P.; MELO, W.O.; MONTEIRO, B.M.; OLIVEIRA, D.R. Desenvolvimento corporal e relação entre biometria e peso de cordeiros lactantes da raça Santa Inês criados na Amazônia. Arquivos Brasileiro de Medicina Veterinária e Zootecnia. v.66, n.6, p.1787-1794, 2014

VARGAS JUNIOR, F.M.; MARTINS, C.F.; SOUZA, C.C.; PINTO, G.S.; PEREIRA, H.F.; CAMILO, F.R.; AZEVEDO JUNIOR, N.P. Avaliação Biométrica de Cordeiros Pantaneiros. Revista Agrarian, v.4, n.11, p.60-65, 2011.
VILLARROEL, A.B.S.; LIMA, L.E.S.; OLIVEIRA, S.M.P.D.; FERNANDES, A.A.O. Ganho de peso e rendimento de carcaça de cordeiros mestiços Texel e Santa Inês x SRD em sistema de manejo semi-intensivo. Ciência Agrotécnica, v.30, n.5, p.971-976, 2006.

YAMAMOTO S. M; A.G.S. SOBRINHO; R.M. VIDOTTI, A. Desempenho e digestibilidade dos nutrientes em cordeiros alimentados com dietas contendo silagem de resíduos de peixe. Revista Brasileira de Zootecnia, v.36, n.4, p.1131-1139, 2007. 